\title{
Non-random Origin of Partial Revertants of a Pleiotropic Phage Resistant Variant of Caulobacter crescentus CB15
}

\author{
By EVELYN T. FARRELL AND PETER R. SMITH \\ Department of Microbiology, University College, Galway, Ireland
}

(Received 27 February 1978)

A variant of Caulobacter crescentus $\mathrm{CB} 15$, strain Gul, was isolated on the basis of its resistance to phage $\phi \mathrm{CbK}$. Strain Gul was also resistant to phages $\phi \mathrm{Cb} 12 \mathrm{r}$ and $\phi \mathrm{Cb} 13$, developed filaments in liquid culture and showed altered sensitivity to the inhibitors methylene blue, sodium deoxycholate and ampicillin. Analysis by sodium dodecyl sulphate-polyacrylamide gel electrophoresis of the membranes of strains Св15 and GU1 showed major differences. Partial revertants of the GUl phenotype were isolated in which only the inhibitor sensitivities were changed. The origin of these partial revertants was investigated by the fluctuation analysis of Luria \& Delbruck (1943). The results suggest that these partial revertants, which were stably inherited, were non-random in origin.

\section{INTRODUCTION}

In attempts to investigate the Caulobacter cell cycle, non-motile, phage resistant and morphological mutants have been isolated. Many of these mutants exhibited pleiotropic changes of phenotype (Fukuda et al., 1976; Kurn \& Shapiro, 1975; Schmidt, 1968). Reversion studies on some of them yielded partial revertants with novel phenotypes which did not occur in mutant or parent cultures (Fukuda et al., 1976; Schmidt, 1968). Similar results have been reported in reversion studies on colicin tolerant mutants in $E$. coli (Rolfe et al., 1974). Gorini \& Beckwith (1966) have argued that the emergence of such novel phenotypic characters indicates that these partial revertants are due to suppressor mutations. Thus, they predict that such revertants would be produced in a random manner. In this paper we report the isolation and partial characterization of a pleiotropic phage resistant mutant of Caulobacter crescentus and the origin of partial revertants of the mutant phenotype.

\section{METHODS}

Strains. All strains were derived from Caulobacter crescentus CB15 which was a gift from Dr L. Shapiro, Albert Einstein College, New York. The peptone yeast extract (PYE) medium of Poindexter (1964) was used throughout. All incubation was carried out at $30{ }^{\circ} \mathrm{C}$ and broth cultures were agitated on a rotary shaker. Phage $\phi \mathrm{CbK}$ was a gift from Dr Shapiro and phages $\phi \mathrm{Cb} 12 \mathrm{r}$ (ATCC 19089-B1) and $\phi$ Cb13 (ATCC 19089-B2) were obtained from the American Type Culture Collection.

Isolation of phage resistant variants. Portions $(0 \cdot 1 \mathrm{ml})$ of $12 \mathrm{~h}$ broth cultures of $\mathrm{CB} 15$ were spread on plates that had been surface-seeded with $10^{9}$ plaque-forming units of phage $\phi \mathrm{CbK}$. The plates were incubated for $48 \mathrm{~h}$ and no more than one colony per plate was subcultured for further testing.

Morphology. All strains were examined for morphological changes by phase-contrast microscopy using a Nikon model L-Ke microscope.

Determination of sensitivity to inhibitors. Sensitivity to ampicillin (Beecham), sodium deoxycholate (BDH) and methylene blue (Gurr, London) was determined by plating approximately 500 colony-forming units (c.f.u.) from a $24 \mathrm{~h}$ broth culture on twofold serial dilutions of the inhibitors in PYE agar. Sensitivity to ampicillin was also determined in PYE broth. The lowest concentration of the drug that inhibited colony 
formation or macroscopically visible turbidity was determined after $48 \mathrm{~h}$ incubation. This concentration was referred to as the minimum inhibitory concentration (m.i.c.).

Isolation of revertants of strain GU1. Variants with decreased sensitivity to methylene blue and sodium deoxycholate were isolated by plating $10^{6}$ to $10^{7}$ c.f.u. from a $24 \mathrm{~h}$ broth culture on twofold serial dilutions of the inhibitors in agar. Colonies which appeared on plates containing the m.i.c. or a higher concentration of the inhibitors were isolated. The alteration of the sensitivities of the putative revertants was checked by plating 500 c.f.u. of strain GU1 and of the variants on identical twofold serial dilutions of the inhibitors in PYE agar.

Fluctuation analysis of reversion in strain GU1. A $12 \mathrm{~h}$ broth culture of bacteria was diluted into $500 \mathrm{ml}$ of prewarmed PYE broth to a final concentration of approximately $10^{2}$ c.f.u. $\mathrm{ml}^{-1}$. Twenty $10 \mathrm{ml}$ portions were distributed to culture tubes and one $200 \mathrm{ml}$ portion was placed in an Erlenmeyer flask. All cultures were incubated until their $A_{660}$ reached $0 \cdot 1$. Cultures which grew at abnormal rates were discarded. Samples $\left(0 \cdot 1 \mathrm{ml}\right.$; approximately $10^{7}$ c.f.u.) of the 20 individual cultures were spread on the surface of selective plates, as were $200.1 \mathrm{ml}$ samples from the bulk culture. Selective plates contained the minimum concentration of the inhibitors that prevented colony formation when inoculated with the strain under test at 500 c.f.u. $\mathrm{ml}^{-1}$. Random colonies were isolated from these selective plates and tested for altered sensitivity to the inhibitors by the method described above.

Phage adsorption. The kinetics of phage adsorption were determined by the method of Schmidt \& Stanier (1965).

Analysis of membrane proteins. Membranes were isolated by the method of Galdiero (1973) and analysed by sodium dodecyl sulphate (SDS)-polyacrylamide gel electrophoresis using the technique of Maizel (1969).

\section{RESULTS}

\section{Origin of strain GU1}

One hundred and fifty phage $\phi \mathrm{CbK}$ resistant variants of $C$. crescentus $\mathrm{CB} 15$ were isolated without the aid of mutagenesis. One hundred and forty-nine of these were also resistant to phages $\phi \mathrm{Cb} 13$ and $\phi \mathrm{Cb} 12 \mathrm{r}$. Of these 149, one strain, GU1, showed altered cell morphology. Approximately $20 \%$ of the cells in PYE broth cultures of GU1 were filaments of 10 to $70 \mu \mathrm{m}$ in length. Cultures of $C$. crescentus CB15 may produce filaments in PYE broth but only do so in old ( 3 to $4 \mathrm{~d}$ ) stationary phase cultures. The filaments produced in old cultures of the wild-type have a characteristic loose spiral morphology (Poindexter, 1964) which is absent from those produced by strain GU1. Strain GU1, on the other hand, is filamentous at all stages of growth. Examination of the membrane proteins of strain GU1 and its parent CB15 by SDS-polyacrylamide gel electrophoresis revealed at least three major differences in band pattern (Fig. 1). Examination of the membrane proteins of strain CB15 grown with sub-inhibitory concentrations of ampicillin or $\mathrm{NaCl}$, conditions which induce filamentation in this strain, showed them to be similar to those of CB15 not GU1. Analysis of the membrane proteins of other variants of $\mathrm{CB} 15$ which were also resistant to $\phi \mathrm{CbK}, \phi \mathrm{Cb} 12 \mathrm{r}$ and $\phi \mathrm{Cb} 13$ showed them to be similar to those of $\mathrm{CB} 15$. This suggests that the changes detected in the protein banding pattern of GU1 are not a necessary consequence of filamentation or of phage resistance.

Strain GU1 also showed altered sensitivity to inhibitors. With respect to ampicillin, strain GU1 was 2.5 times more resistant than $\mathrm{CB} 15$ in solid medium and twice as sensitive in liquid medium. With respect to sodium deoxycholate and methylene blue in solid media, strain GU1 was twice as sensitive as the parent strain св15. The reproducibility of twofold differences in sensitivity to inhibitors was tested by carrying out 20 independent determinations of the relative sensitivity of $\mathrm{CB} 15$ and GU1 to the inhibitors. The reproducibility was $100 \%$ with respect to ampicillin and $90 \%$ with respect to methylene blue and deoxycholate. Thus, these changes in sensitivity cannot be artefacts of the test procedure. The phenotype of GU1 (Table 1) has remained constant over 4 years storage, culturing and testing. 

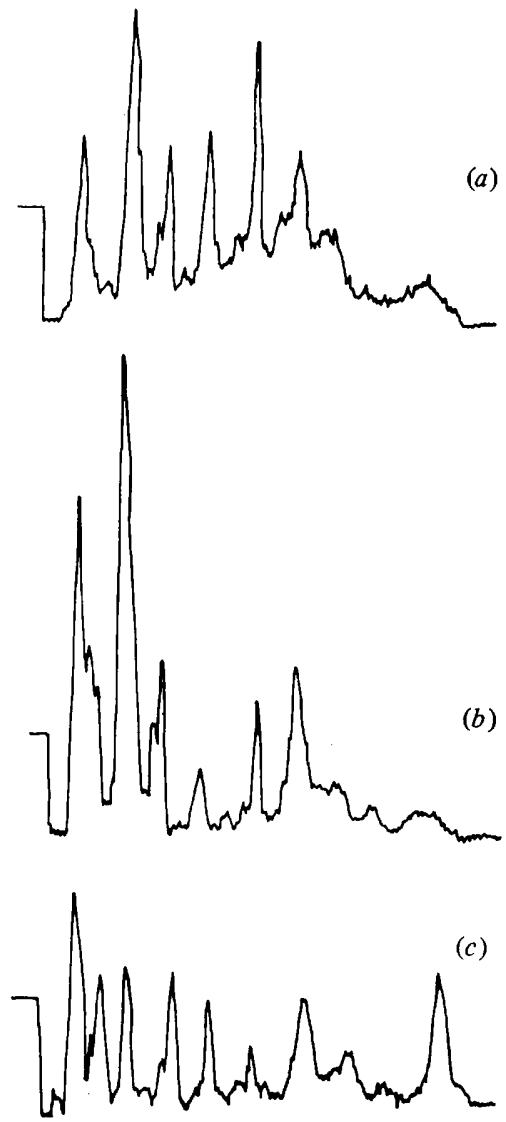

Fig. 1. Densitometer tracings of membrane protein patterns on SDS-polyacrylamide gel electrophoresis of Caulobacter crescentus. (a) Strain CB15 grown in PYE with ampicillin $\left(150 \mu \mathrm{g} \mathrm{ml}^{-1}\right)$. (b) Strain CB15 grown in PYE. (c) Strain GU1 grown in PYE. Revertants of strain GU1 showed patterns that were identical with that from Gul.

Table 1. Phenotypes of C. crescentus strains CB15 and Gu1

\section{Phenotype}

$\phi \mathrm{CbK}$

$\phi \mathrm{Cb} 12 \mathrm{r}$

$\phi \mathrm{Cb} 13$

$50 \% \phi \mathrm{CbK}$

adsorption

Filaments

Ampicillin in broth*

Ampicillin agar*

Deoxycholate*

Methylene blue*
Strain CB15

Sensitive

Sensitive

Sensitive

35 min

Absent

400

300

1000

0.5
Strain GU1

Resistant

Resistant

Resistant

$100 \mathrm{~min}$

Present

200

750

500

$0 \cdot 25$

* Figures refer to minimum inhibitory concentrations of inhibitors in $\mu \mathrm{g} \mathrm{ml}^{-1}$. 
Table 2. Distribution of the numbers of resistant bacteria in 20 samples of cultures of $C$. crescentus strains $\mathrm{CB} 15$ and $\mathrm{GU} 1$

\begin{tabular}{lllrrr} 
& & & \multicolumn{3}{c}{ Selective agent $\dagger$} \\
\cline { 3 - 5 } Strain & Source & & MB & DOC & KAN \\
CB15 & Same culture & Mean $\bar{x}$ & 288 & 122 & 70 \\
& & Variance $V^{*}$ & 1374 & 173 & 186 \\
& & $V / \bar{x}$ & $4 \cdot 7$ & $1 \cdot 4$ & $2 \cdot 6$ \\
CB15 & Similar cultures & Mean $\bar{x}$ & 308 & 45 & 324 \\
& & Variance $V^{*}$ & 14846 & 1757 & 8147 \\
& & $V / \bar{x}$ & $48 \cdot 2$ & 39 & 25 \\
GU1 & Same culture & Mean $\bar{x}$ & 92 & 72 & 109 \\
& & Variance $V^{*}$ & 52 & 145 & 259 \\
& & $V / \bar{x}$ & 0.57 & $2 \cdot 0$ & $2 \cdot 3$
\end{tabular}

* Corrected for sampling error (Luria \& Delbruck, 1943).

$\dagger \mathrm{MB}$, Methylene blue; DOC, sodium deoxycholate; KAN, kanamycin.

\section{Fluctuation analysis of partial revertants of strain GU1}

When dense cultures of strain Gul were plated on agar containing the m.i.c. of methylene blue (MB) and sodium deoxycholate (DOC) for that strain, colonies were formed by approximately $1 \times 10^{-6}$ and $5 \times 10^{-5}$ of the total c.f.u. plated, respectively. To shed more light on the origin and nature of these revertants the frequency of their occurrence was analysed by the fluctuation test of Luria \& Delbruck (1943). Table 2 shows the results of plating 20 samples from a single $200 \mathrm{ml}$ culture of GUl and of CB15 on agar containing their respective minimum inhibitory concentrations of $\mathrm{MB}, \mathrm{DOC}$ and kanamycin. With both strains the variance between the numbers of clones in the series of samples capable of forming colonies was close to their mean value indicating that the variation, as would be expected, is a result of chance. Table 2 also presents the results of plating samples of 20 independent cultures of strain $\mathbf{C B} 15$ on agar containing the minimum inhibitory concentrations of the same drugs. In these cases the numbers of clones in each culture capable of forming colonies showed a variance much larger than their mean as would be predicted by the theory of random mutation. Table 3 presents the results and analysis of plating samples of 20 independent cultures of strain GUl on agar containing the minimum inhibitory concentrations of MB, DOC and kanamycin. The distribution of colonies on agar containing kanamycin showed a high variance but the distributions on agar containing MB and DOC did not.

Variants of strain Gul that formed colonies on agar containing the minimum inhibitory concentrations of $\mathrm{MB}$ and DOC were isolated. Fifty independent variants were selected in this way from each selective medium, and their phenotypes were analysed (Table 4). None of these 100 strains showed gain of phage $\phi \mathrm{CbK}$ sensitivity, loss of filamentation or reversion to wild-type CB15 SDS-polyacrylamide gel electrophoresis membrane protein bands. With respect to ampicillin sensitivity, $26 \%$ of variants selected on MB agar had a wild-type СB 15 level of resistance and $28 \%$ of the variants selected on DOC agar had this phenotype. The phenotypes shown in Table 4 for these partial revertants were stable during a minimum of four cycles of plating, subculturing and testing. Microscopic examination of broth cultures of variants of strain GU1 selected on MB agar showed that $20 \%$ of them contained deformed cells termed 'fats'. No 'fats' were seen in cultures of variants selected on DOC agar or in any other cultures of GU1. In contrast, variants of strain CB 15 selected for a twofold increase in resistance to MB or DOC showed no unselected phenotypic changes. 
Table 3. Distribution of the numbers of resistant bacteria in a series of similar cultures of $C$. crescentus Gul

Selective agent $\uparrow$

\begin{tabular}{|c|c|c|c|c|c|c|c|c|c|c|c|c|}
\hline \multirow[b]{2}{*}{$\begin{array}{l}\text { No. of } \\
\text { cultures ... }\end{array}$} & \multicolumn{4}{|c|}{ MB } & \multicolumn{4}{|c|}{ DOC } & \multicolumn{4}{|c|}{$\mathrm{KAN}^{\mathrm{KAN}}$} \\
\hline & 20 & 20 & 17 & 14 & 19 & 18 & 20 & 16 & 20 & 18 & 20 & 20 \\
\hline $\begin{array}{l}\text { Culture no. } \\
1\end{array}$ & 5 & 10 & 11 & 46 & 290 & 78 & 100 & 106 & 93 & 162 & 225 & 21 \\
\hline 2 & 12 & 2 & 12 & 34 & 310 & 89 & 95 & 72 & 69 & $\begin{array}{r}102 \\
89\end{array}$ & 314 & 54 \\
\hline 3 & 0 & 11 & 28 & 52 & 236 & 54 & 79 & 73 & 101 & 78 & 345 & 90 \\
\hline 4 & 10 & 6 & 22 & 36 & 226 & 66 & 107 & 61 & 116 & 130 & 190 & 71 \\
\hline 5 & 8 & 15 & 20 & 28 & 259 & 68 & 76 & 80 & 205 & 86 & 452 & 62 \\
\hline 6 & 6 & 8 & 48 & 48 & 288 & 71 & 131 & 67 & 81 & 100 & 294 & 397 \\
\hline 7 & 8 & 6 & 33 & 28 & 243 & 56 & 108 & 92 & 180 & 69 & 250 & 108 \\
\hline 8 & 14 & 7 & 47 & 24 & 324 & 62 & 89 & 52 & 135 & 67 & 220 & 27 \\
\hline 9 & 6 & 33 & 13 & 31 & 284 & 77 & 114 & 57 & 346 & 72 & 326 & 108 \\
\hline 10 & 7 & 6 & 43 & 26 & 271 & 89 & 119 & 90 & 100 & 90 & 480 & 131 \\
\hline 11 & 9 & 12 & 28 & 35 & 292 & 62 & 100 & 73 & 91 & 384 & 390 & 136 \\
\hline 12 & 11 & 15 & 29 & 34 & 256 & 70 & 80 & 78 & 85 & 157 & 188 & 27 \\
\hline 13 & 6 & 9 & 26 & 30 & 258 & 67 & 82 & 50 & 100 & 163 & 374 & 204 \\
\hline 14 & 6 & 5 & 33 & 34 & 253 & 79 & 83 & 56 & 150 & 91 & 302 & 181 \\
\hline 15 & 13 & 20 & 29 & & 213 & 77 & 85 & 51 & 67 & 66 & 492 & 125 \\
\hline 16 & 8 & 12 & 40 & & 244 & 92 & 83 & 65 & 109 & 52 & 392 & 186 \\
\hline 17 & 12 & 5 & 23 & & 270 & 97 & 114 & & 112 & 78 & 294 & 71 \\
\hline 18 & 20 & 10 & & & 294 & 53 & 109 & & 136 & 480 & 258 & 81 \\
\hline 19 & 5 & 8 & & & 321 & & 72 & & 45 & & 286 & 179 \\
\hline 20 & 12 & 18 & & & & & 102 & & 106 & & 421 & 185 \\
\hline Mean $\bar{x}$ & 8.9 & $10 \cdot 9$ & $28 \cdot 5$ & $34 \cdot 7$ & 275 & 81 & 101 & 70 & 121 & 131 & 324 & 122 \\
\hline$V^{*}$ & $8 \cdot 6$ & 35 & 95 & 32 & 1077 & 283 & 530 & 201 & 14641 & 12213 & 8147 & 7037 \\
\hline$V / \bar{x}$ & 0.96 & $3 \cdot 2$ & $3 \cdot 3$ & 0.93 & $3 \cdot 9$ & $3 \cdot 4$ & $5 \cdot 2$ & $2 \cdot 8$ & 33 & $93 \cdot 2$ & 25 & $57 \cdot 6$ \\
\hline
\end{tabular}

* Corrected for sampling error (Luria \& Delbruck, 1943).

$\uparrow$ MB, Methylene blue; DOC, sodium deoxycholate; KAN, kanamycin.

\section{Table 4. Phenotypes of partial revertants of C. crescentus strain Gu1}

Phenotype characters tested were: sensitivity to $\phi \mathrm{CbK}, \phi \mathrm{Cb} 12 \mathrm{r}$ and $\phi \mathrm{Cb} 13$; SDS-polyacrylamide gel electrophoresis membrane protein patterns; filamentation; sensitivity to methylene blue (MB), sodium deoxycholate (DOC) and ampicillin (AMP). Characters unchanged from strain GU1 are not shown; characters changed to wild-type св15 levels are indicated by capitals; characters changed to at least twofold higher resistance than wild-type св 15 are indicated by capitals followed by a plus sign.

\begin{tabular}{lcc}
\multicolumn{1}{c}{ Phenotype } & $\begin{array}{c}\text { Pelectentage of isolates of each } \\
\text { phenotype }\end{array}$ \\
DOC & - & $\begin{array}{c}\text { Selected on DoC } \\
\text { agar }\end{array}$ \\
DOC+ & - & 14 \\
MB & 26 & 6 \\
DOC, MB & 22 & 18 \\
DOC+, MB & 26 & 20 \\
DOC, MB+ & - & 4 \\
DOC+, MB+ & - & 10 \\
DOC, AMP & - & 2 \\
MB, AMP & 4 & 26 \\
DOC, MB, AMP & 10 & - \\
DOC+, MB, AMP & 8 & - \\
DOC, MB+, AMP & 4 &
\end{tabular}




\section{DISCUSSION}

Strain GU1 has maintained a different phenotype from that of its parent св15 through numerous subcultures during 4 years. Thus, its phenotypic change must be considered as a stable inherited change. Similar phenotypic changes have been reported for class II amp mutants in E. coli (Nordstrom et al., 1970). These mutants show poor adsorption of phages $\mathrm{T} 3$ and $\mathrm{T} 4$, small variations in sensitivity to a variety of inhibitors, a twofold increase in resistance to ampicillin in solid media and increased sensitivity to ampicillin in broth media. These phenotypic changes have, in some mutants, been shown to be the consequences of mutations in $\operatorname{lps} A$ or $\operatorname{lps} B$ or galU genes, which lead to the production of an altered lipopolysaccharide.

No evidence is presented in this paper as to the causal lesions involved in the change of phenotypes from the wild-type strain CB 15 to the variant GU1. The phenotypes of the partial revertants of GU1 do suggest that the control of the phenotypic characters of phage resistance, filament formation and membrane protein patterns, to which unselected phenotypic variants were not detected in the 100 strains tested, may be under a different control system from those of MB, DOC and ampicillin sensitivity. Unselected phenotypic variants were isolated in these latter properties in 78,70 and $26 \%$ of the strains tested (Table 4). These partial revertants showed reproducible phenotypic changes which were stably inherited through many generations.

Luria \& Delbruck (1943) predicted that if a particular phenotypic change was the result of an adaptation mechanism, then the distribution of the changed cells in a number of similar cultures would show a variance equal to the mean. If, on the other hand, such changes were caused by random genetic changes, then the variance would be 'abnormally high'. They further stated that this prediction would not be affected by the number of different mutational events that could give rise to the phenotypic change that was being selected. The essential condition for this prediction is that the initial number of bacteria in a culture must be so small that the number of mutations which occur during the first division cycle of the bacteria is small. This condition has been met in the experiments reported here where the initial number was approximately $10^{2}$ c.f.u. per culture.

The results for strain Gul (Table 3) show that the distribution of revertants, i.e. cells capable of colony formation on plates containing the m.i.c. of MB and DOC, in a series of similar cultures do not show the predicted abnormally high variance. The variance is, in fact, close to the mean. These results are not consistent with the predictions of an explanation of the origin of the partial revertants by random mutation. On the other hand, the distribution of cells in a series of similar cultures of св15 capable of forming colonies on plates containing the m.i.c. for CB15 of MB and DOC does show an abnormally high variance. Thus, the failure to demonstrate a high variance for GU1 cannot be explained by reference to either the particular selective agents used or to the smallness of the twofold increase in resistance being selected. These conditions are identical in the experiments both with CB15 and with GU1. The distribution of cells in a series of cultures of strain GU1 capable of growing on agar containing the m.i.c. of kanamycin shows the high variance predicted by the random mutation theory. Thus, it is not possible to explain the low variance in the distribution of colonies of GUl on agar containing MB and DOC by reference to some unknown property of strain GUl. This work suggests that the MB and DOC resistant variants of strain GU1, but not those of СB 15 , are produced by a mechanism other than that by which normal mutants originate. It is interesting that the GU1 variants produced by this abnormal mechanism show pleiotropic changes whereas the $\mathrm{CB} 15$ variants produced by a mechanism consistent with the random mutation theory showed no unselected pleiotropic changes.

The most reasonable conclusion is that the partial revertants represent the result of an adaptive inheritable change in GU1. Such a change may theoretically be inherited either in 
the genotype or phenotype. Our current understanding of the flow of information in cells makes the hypothesis of adaptive changes in genetic information unattractive. The alternative of adaptive changes in phenotypes that are inheritable thus appears to be the most plausible explanation of the partial revertants. A similar hypothesis has previously been suggested to explain the development of heat resistance in Streptococcus faecium recovering from heat shock (Duitschaever \& Jordan, 1974). It may not be a trivial coincidence that, in both the work reported here and in the work on S. faecium, the most probable site of the phenotypic change is in the membrane of the cell.

\section{REFERENCES}

Duitschaever, C. L. \& JoRdan, D. C. (1974). Development of resistance to heat and sodium chloride in Streptococcus faecium recovering from thermal injury. Journal of Milk and Food Technology 37, 382-386.

FukUda, A., MiYakawa, K., IIDA, H. \& OKada, Y. (1976). Regulation of polar surface structures in Caulobacter crescentus: pleiotropic mutations affect the coordinate morphogenesis of flagella, pili and phage receptors. Molecular and General Genetics 149, 167-173.

Galdiero, F. (1973). The growth and partition of cell membranes during synchronized division cycle of Caulobacter crescentus. Archiv für Mikrobiologie 94, 125-133.

GoRINI, L. \& BeCKWITH, J. (1966). Suppression. Annual Review of Microbiology 20, 401-422.

KuRN, N. \& Shapiro, L. (1975). Regulation of the Caulobacter cell cycle. In Current Topics in Cellular Regulation, vol. 9, pp. 41-64. Edited by B. L. Horecker \& E. R. Stadtman. San Francisco: Academic Press.

LuRIA, S. E. \& Delbruck, M. (1943). Mutations of bacteria from virus sensitivity to virus resistance. Genetics 28, 491-511.
Maizel, J. V. (1969). Acrylamide gel electrophoresis of proteins and nucleic acids. In Fundamental Techniques in Virology, pp. 334-362. Edited by K. Habel \& N. S. Salzman. New York: Academic Press.

Nordstrom, K., Burman, L. G. \& EriksonGREENBURG, K. G. (1970). Resistance of Escherichia coli to penicillins. VII. Physiology of a class II ampicillin resistant mutant. Journal of Bacteriology 101, 659-668.

PoindeXter, J. K. S. (1964). Biological properties and classification of the Caulobacter group. Bacteriological Reviews 28, 231-295.

Rolfe, B., Creaser, E. H. \& Schell, J. (1974). A colicin tolerant mutant of $E$. coli with reduced levels of cAMP and the regulation of lambdoid phage lysogeny. Molecular and General Genetics 130, 105-113.

SCHMidT, J. M. (1968). Stalk elongation in mutants of Caulobacter crescentus. Journal of General Microbiology 53, 291-298.

SCHMidT, J. M. \& STANIER, R. Y. (1965). Isolation and characterization of bacteriophages active against stalked bacteria. Journal of General Microbiology 39, 95-107. 